\title{
Extension of the IGS Repro3 ANTEX file with BeiDou and QZSS satellite antenna pattern
}

\author{
A. Villiger, R. Dach, L. Prange, A. Jäggi \\ Astronomical Institute, University of Bern, Switzerland \\ Contact:arturo.villiger@aiub.unibe.ch
}

EGU General Assembly 2021

23. April 2021, Vienna, Austria 


\section{Content}

\section{Goal:}

Test disclosed GPS BLOCK IIIA, BEIDOU, and QZSS PCOs w.r.t. the ICS Repro3 ANTEX (based on Galileo scale)

Test:

Phase Center Offset (PCO) / Phase Variations (PV) estimation constraining Galileo pattern using multi-GNSS receiver antenna calibrations (IGSR3 ANTEX file) based on CODE MGEX processing scheme

\section{Outcome:}

GPS BLOCK III compatible (except of G74)

BeiDou could be added (as a non-official extension of the Repro3 ANTEX) 


\section{Analysed satellites}

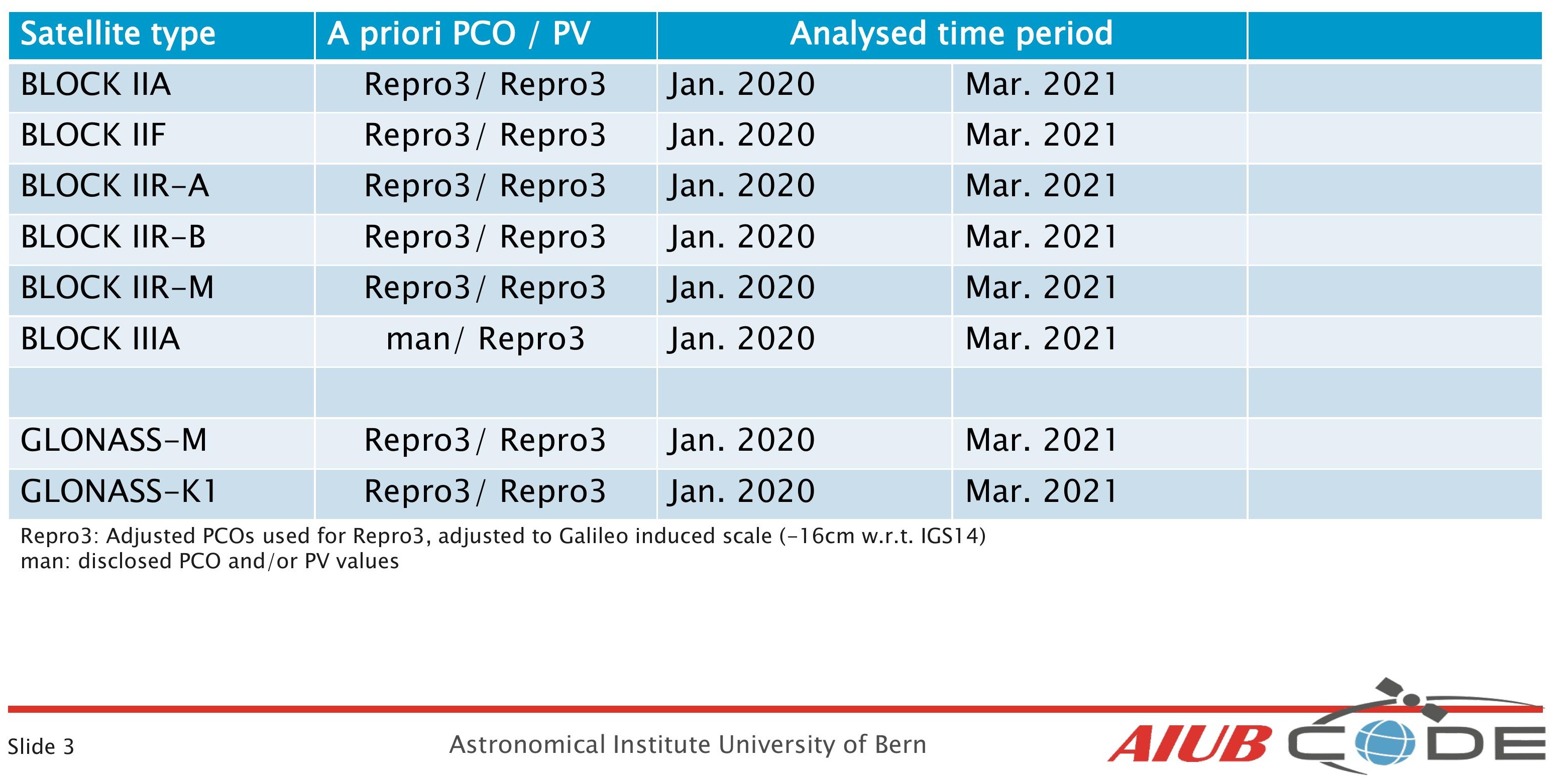




\section{Analysed satellites}

\begin{tabular}{|l|c|l|l|}
\hline Satellite type & A priori PCO / PV & \multicolumn{2}{|c|}{ Analyzed time period } \\
\hline BEIDOU 2I & man / - & Jan. 2020 & Mar. 2021 \\
\hline BEIDOU 2M & man / - & Jan. 2020 & Mar. 2021 \\
\hline BEIDOU 3SI SECM & man / - & Jan. 2021 & Mar. 2021 \\
\hline BEIDOU 3SI CAST & man / - & Jan. 2021 & Mar. 2021 \\
\hline QZSS & man / man & Jan. 2020 & Mar. 2021 \\
\hline Galileo IOV & man / man & constrained & \\
\hline Galileo FOC & man / man & constrained & \\
\hline
\end{tabular}

R20: Adjusted PCOs used for Repro3, adjusted to Galileo induced scale (ca. $-16 \mathrm{~cm}$ w.r.t. IGS14) man: disclosed PCO and/or PV values 


\section{System-wise z-PCO difference w.r.t. a priori information}

\begin{tabular}{|l|c|c|}
\hline GNSS & A priori & $\begin{array}{c}\text { Receiver: } \\
\text { IGSR3 }\end{array}$ \\
\hline GPS & Repro3 & $-0.3 \mathrm{~cm}$ \\
\hline GLONASS & Repro3 & $-0.3 \mathrm{~cm}$ \\
\hline Galileo & man & - \\
\hline BeiDou & man & $+50 \mathrm{~cm}$ \\
\hline QZSS & man & $-182 \mathrm{~cm}$ \\
\hline
\end{tabular}

\section{Setup:}

The normal equations of one day solutions covering 2020-2021 have been combined and a system-wise GNSS offset was setup. The estimated offset is applied to each satellite on top of their a priori values.

\section{GPS and GLONASS:}

For those two system the offset are almost 0 . This is expected as their PCOs have been adjusted in the Repro3 ANTEX to be compatible with the Galileo induced scale.

\section{QZSS}

Our estimated Z-PCO difference seems to be rather high. However, one should keep in mind that those are QZS satellites and due to the geometry the PCO have a smaller influence on the station height.

\section{BeiDou}

The system wise correction for BeiDou is $50 \mathrm{~cm}$. Note that the offset is a combined difference for all BeiDou satellites including IGSO satellites (GEO satellites are not processed).

For BeiDou the system-wise correction seems not to be appropriate to test the disclosed pattern.

In the next slides individual PCO estimations will be presented. 


\section{GPS: satellite-wise PCO corrections}

\section{Z-PCO}

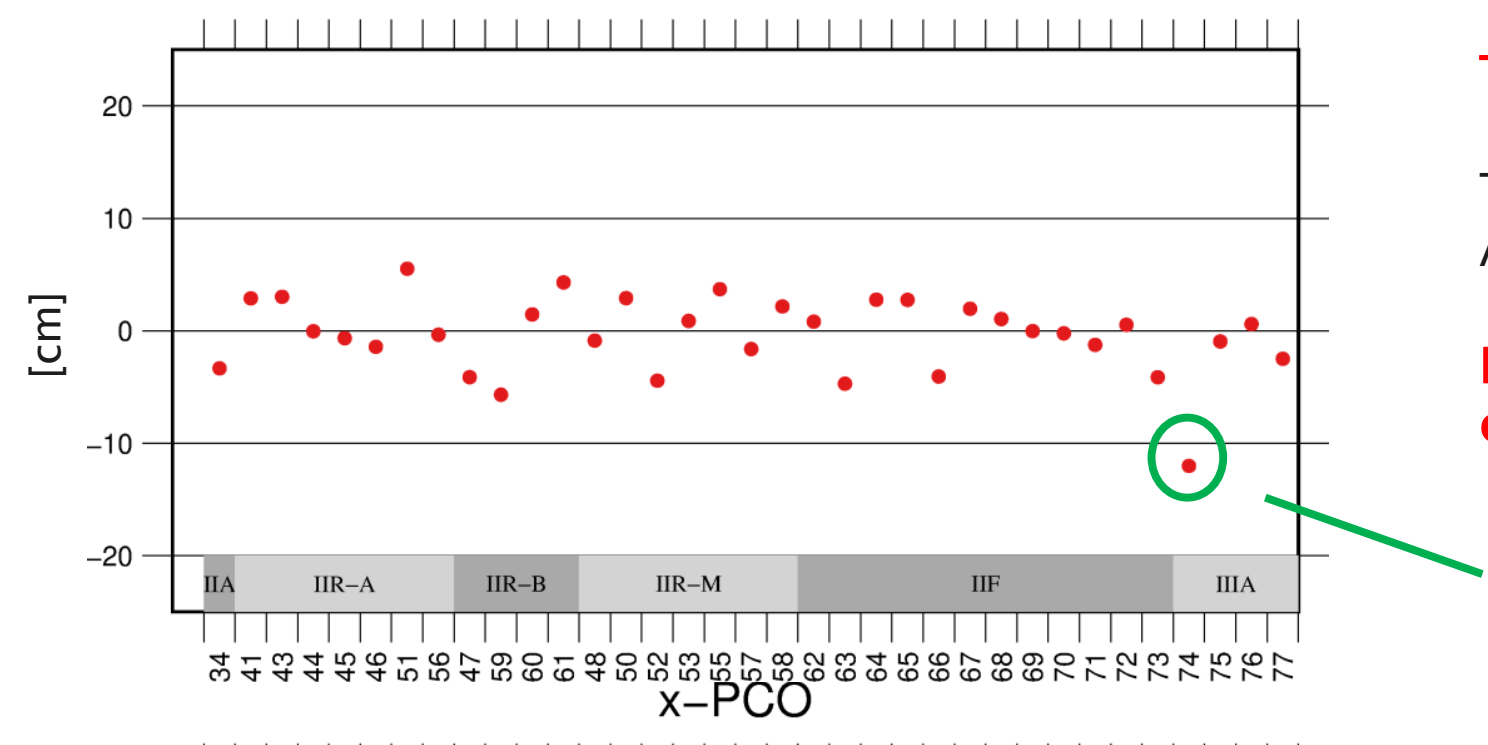

The average PCOs are around 0 which is to be expected.

The used a priori PCOs are form the IGSR3 ANTEX files which Are aligned to the Galileo induced scale

Recommendation: Disclosed BLOCK IIIA PCO values could be used for future ITRF solution (except of G074)

Disclosed BLOCK IIIA SVN74 Z-PCO value does not seem to fit compared to the later launched BLOCK IIIA satellites

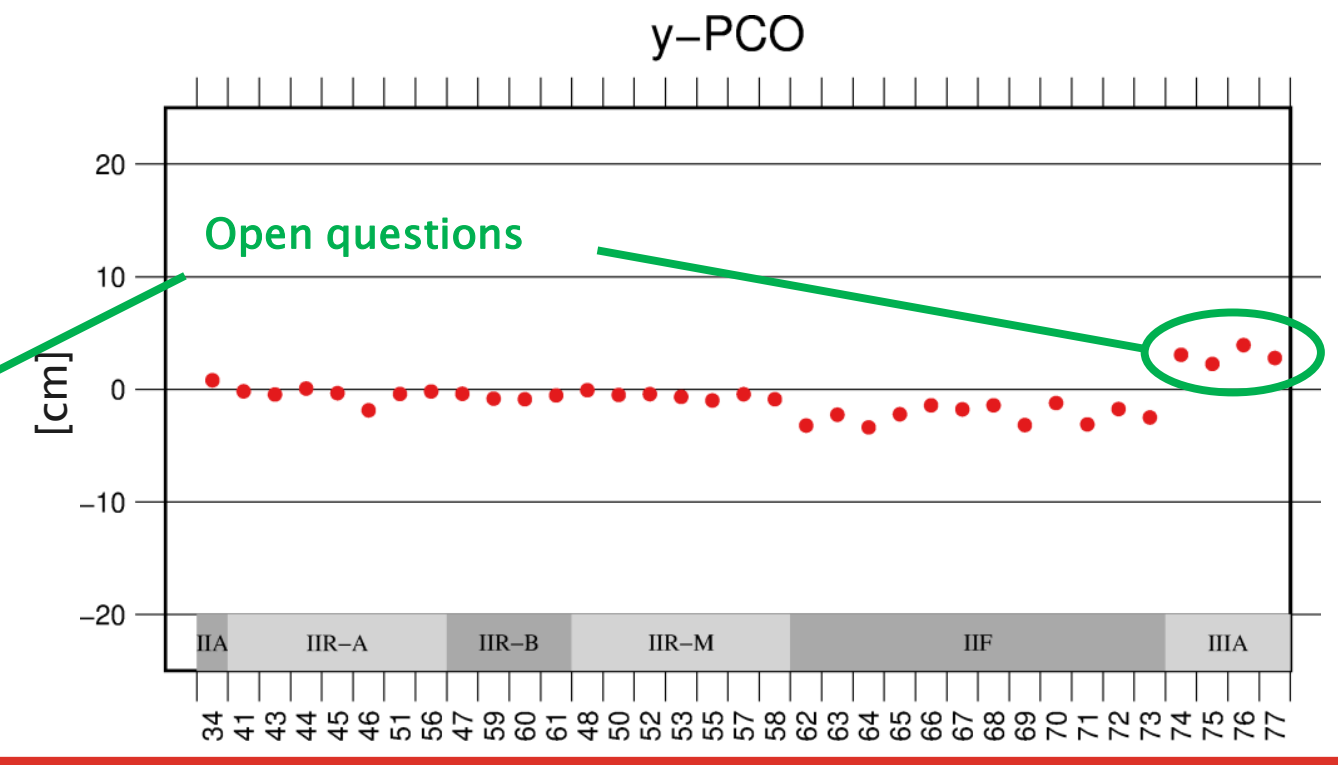

Average RMS: Z-PCO: $0.5 \mathrm{~cm}$ $\mathrm{X}-\mathrm{PCO}: 0.3 \mathrm{~cm}$ y-PCO: $0.3 \mathrm{~cm}$ 


\section{GPS: Phase Variations}

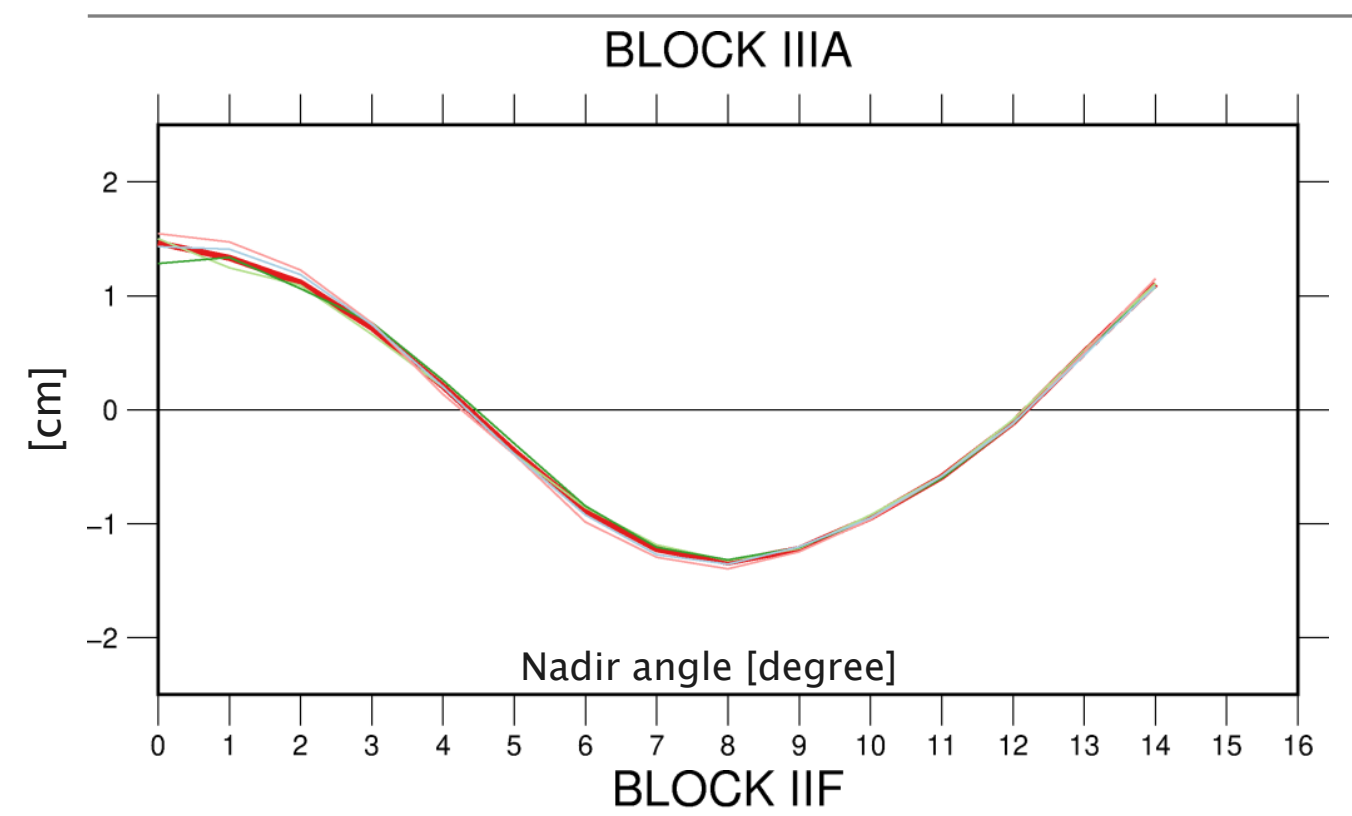

\section{Solutions:}

Two different solution are estimated:

- A common nadir dependent PV set for each satellite type

- Individual PV pattern for each satellites

\section{Results:}

The IGS is using block specific nadir dependant phase variations for the satellites. This is also applicable for the latest GPS satellite generation (BLOCK IIIA)
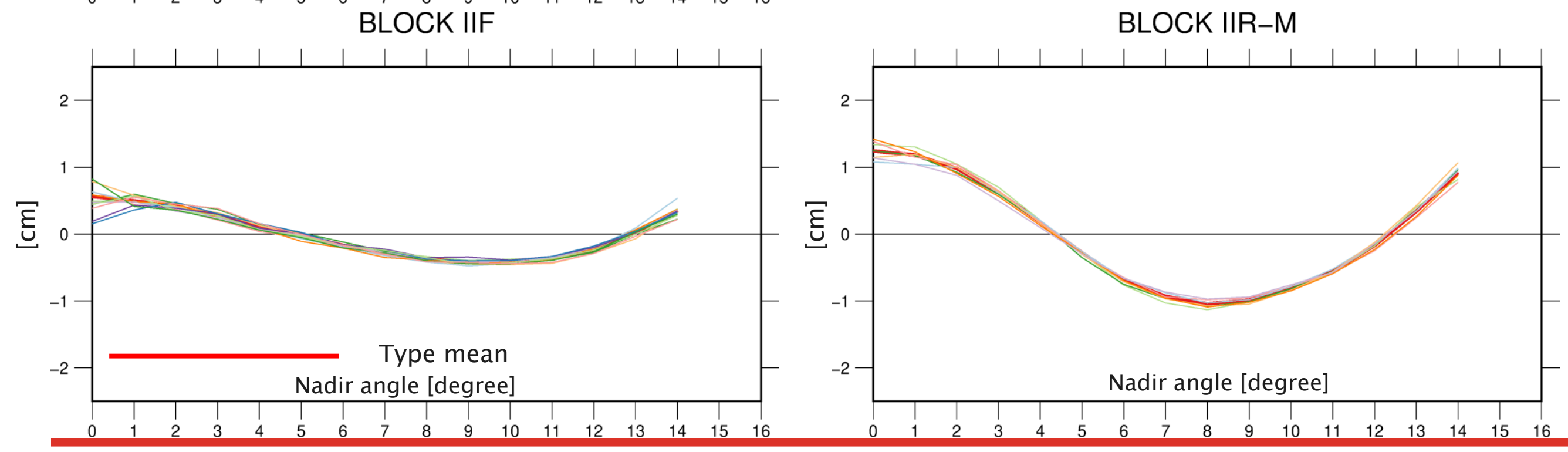


\section{GLONASS: satellite-wise PCO corrections}

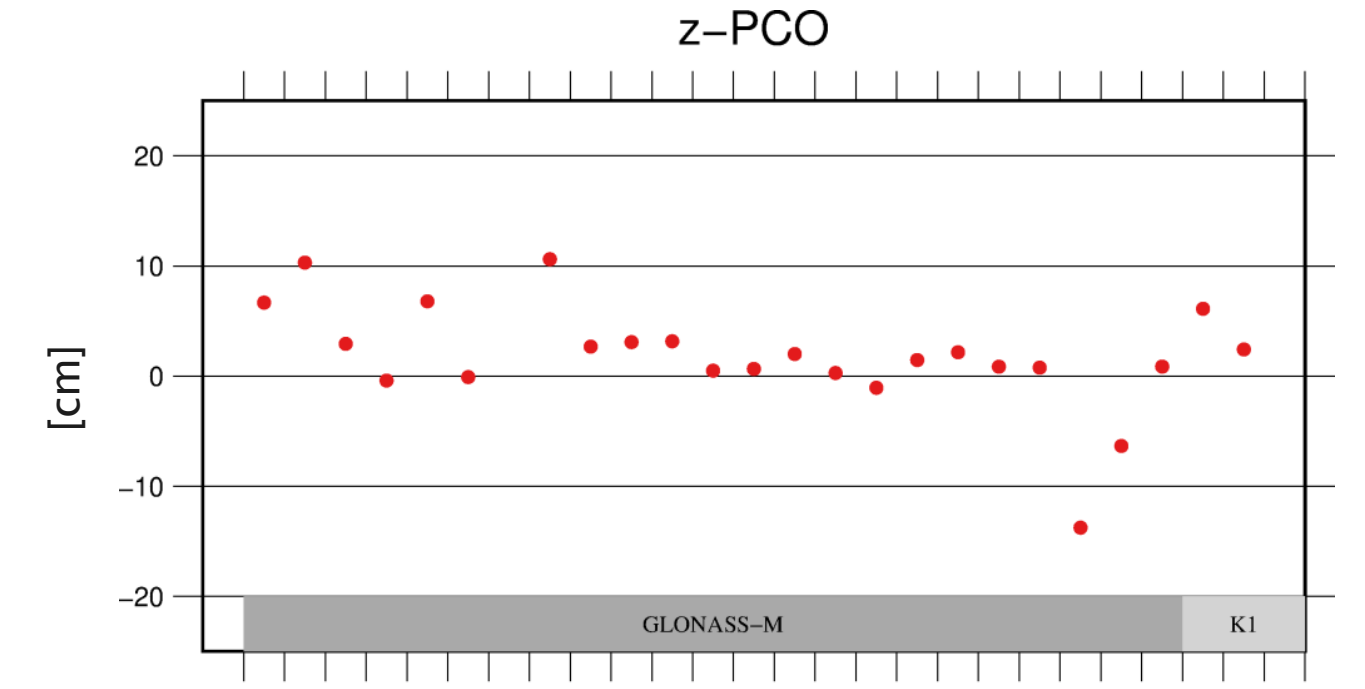

The average PCO corrections are around 0 which is to be expected.

The used a priori PCOs are from the ICSR3 ANTEX file which are aligned to the Galileo induced scale

Outliers: R733 (z: $-4.43 m+/-2,5 m)$ and R805 (z: $1.55 m+/-5 \mathrm{~cm})$ are not shown in z-PCO plot

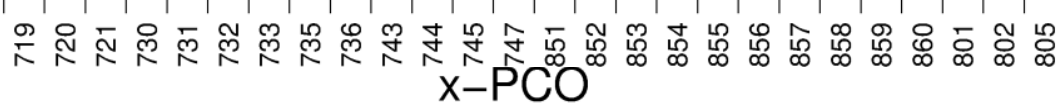

$$
\text { y-PCO }
$$

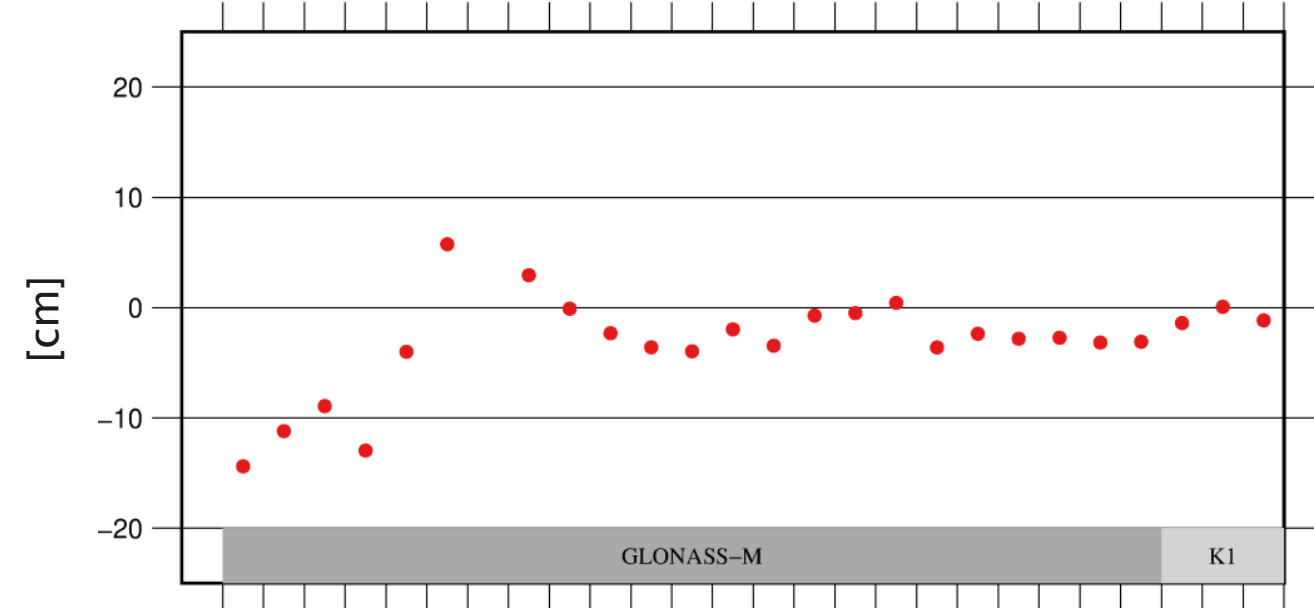

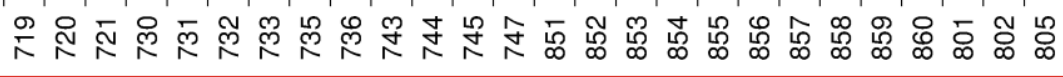

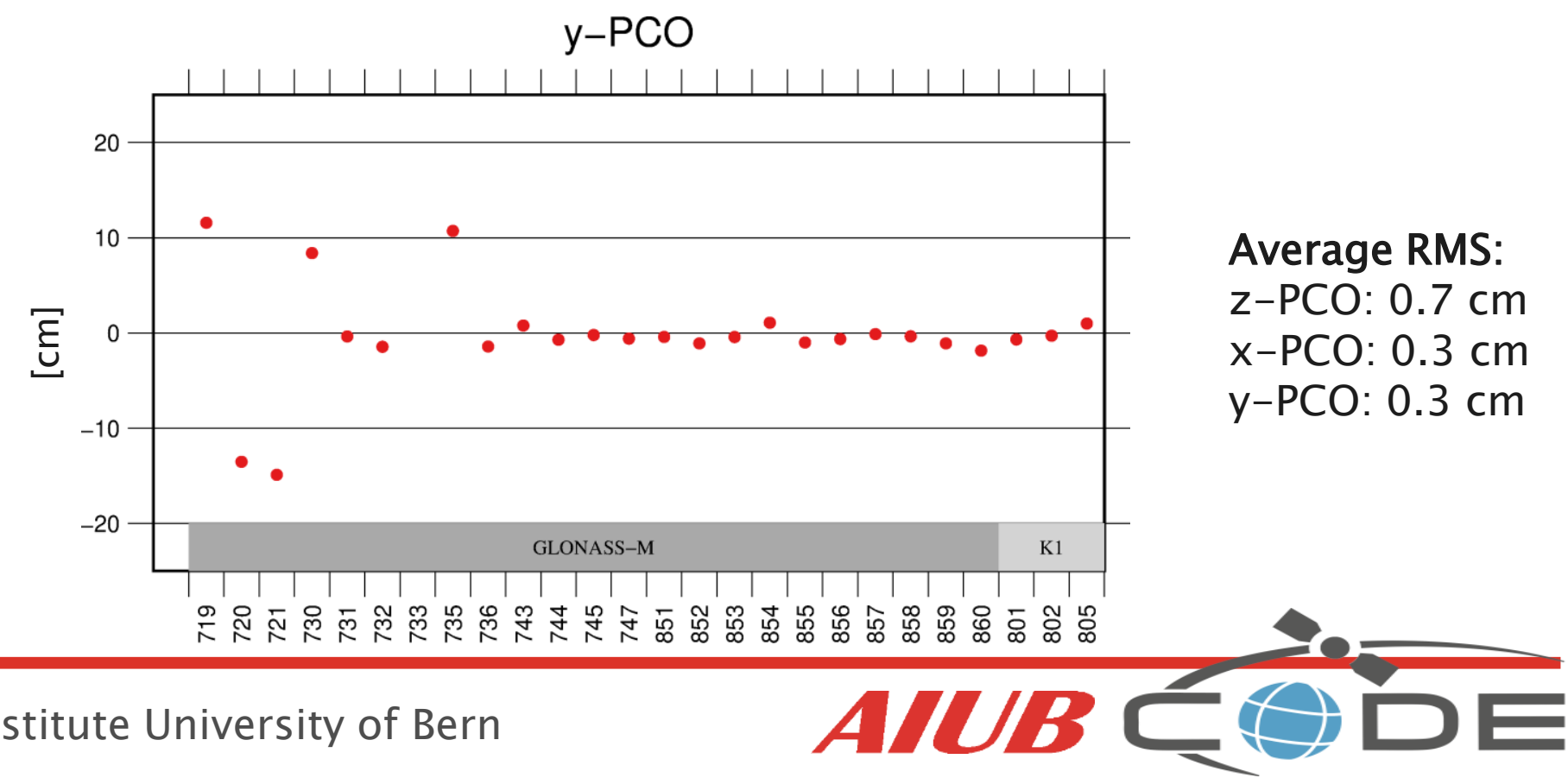




\section{GLONASS: Phase variations}

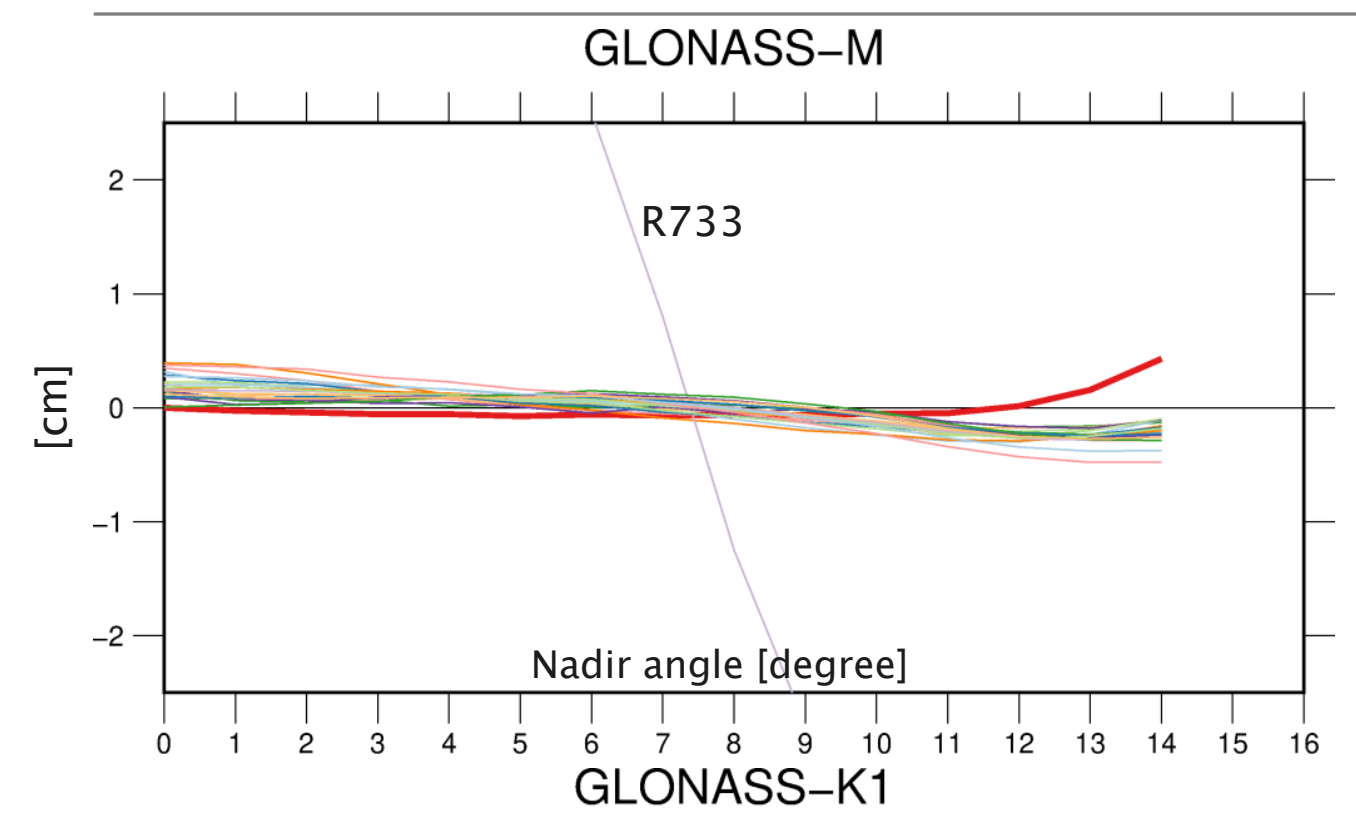

\section{Solutions:}

Two different solution are estimated:

- A common nadir dependent PV set for each satellite type

- Individual PV pattern for each satellites

\section{Results:}

The IGS is using block specific nadir dependant phase variations for the Satellites.

\section{Outliers:}

The same satellites which have questionable PCO estimations show the same behaviour in in their PV patterns.

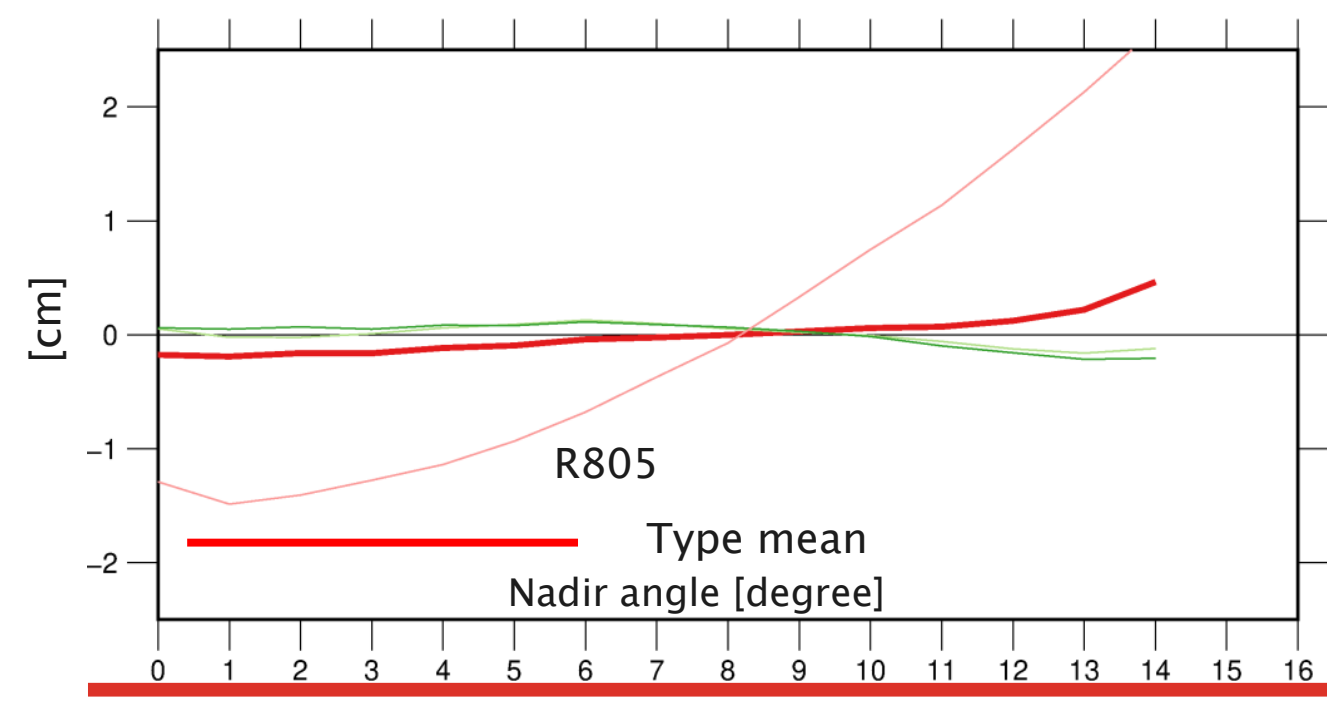

\section{Cause:}

The satellites are only observed for a short time period and thus not well observed. 


\section{BeiDou (PCO corrections)}

z-PCO

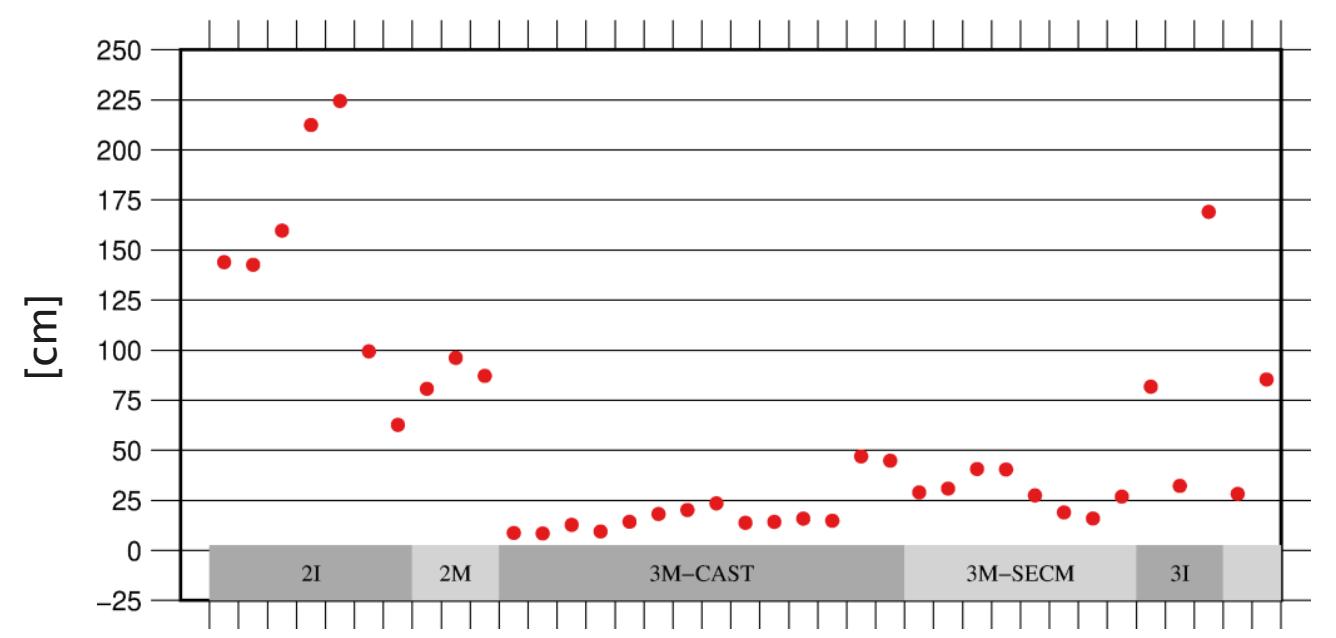

x-PCO

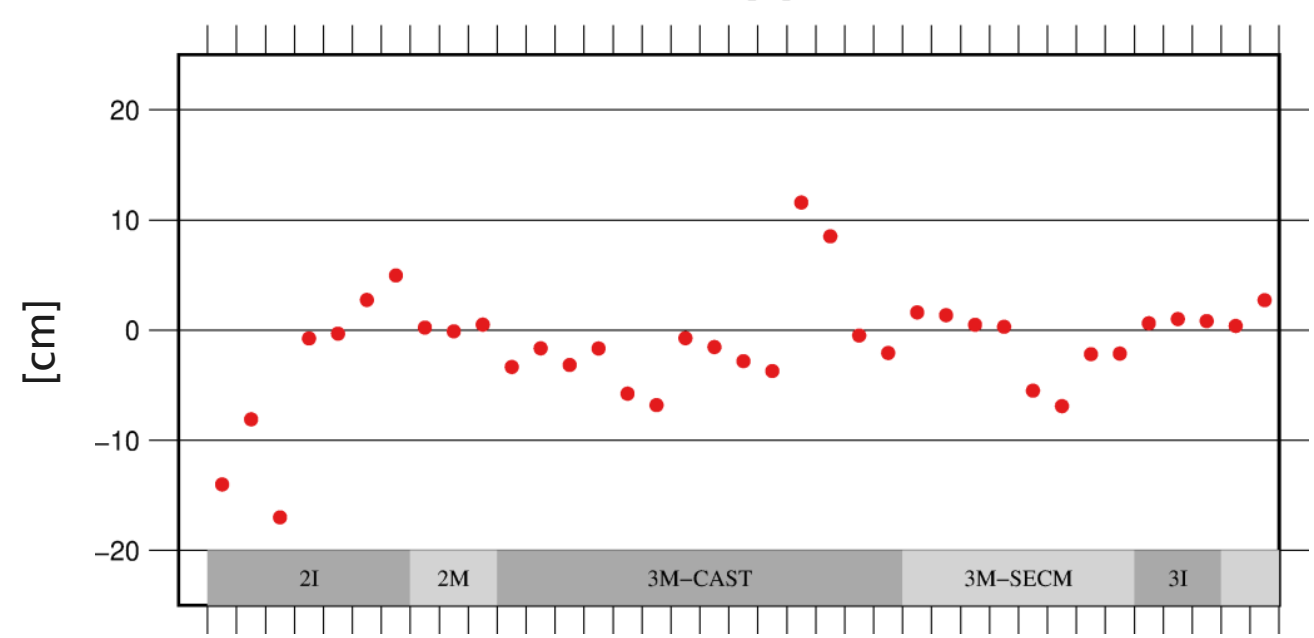

\section{IGSO satellites:}

The station heights are less sensitive to the zPCOs of the ICSO compared to the MEO satellites. Different studies showed quite some differences for the IGSO estimates.

\section{MEO satellites}

The BDS-3 satellites have an offset of about $10 \mathrm{~cm}$. The values could be added (as BeiDou is not part of the official Repro3) to the Repro3 ANTEX. Further studies are needed for a potential future contribution to an ITRF

$$
y-P C O
$$

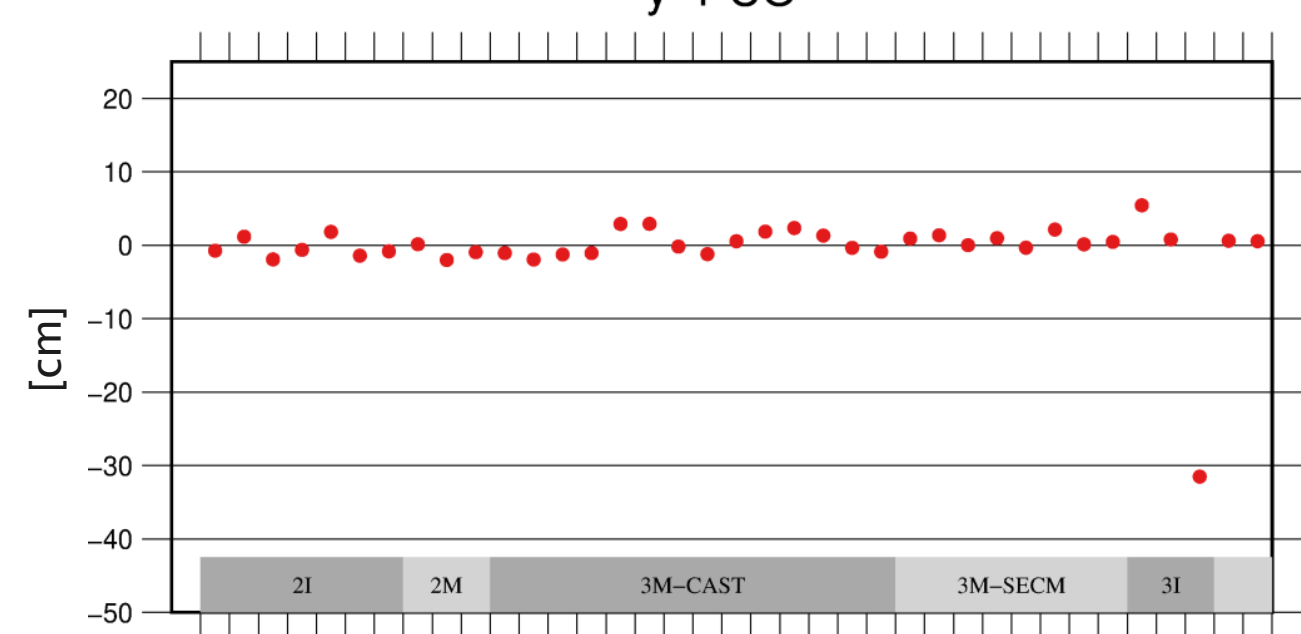

Average RMS: BDS 2:

Z-PCO: $2 \mathrm{~cm}$ $X$-PCO: $0.8 \mathrm{~cm}$ y-PCO: $0.5 \mathrm{~cm}$ BDS 3M:

Z-PCO: $2 \mathrm{~cm}$ $\mathrm{X}-\mathrm{PCO}: 0.8 \mathrm{~cm}$ y-PCO: $0.5 \mathrm{~cm}$ 


\section{Inter system translation bias (GPS-Beidou)}
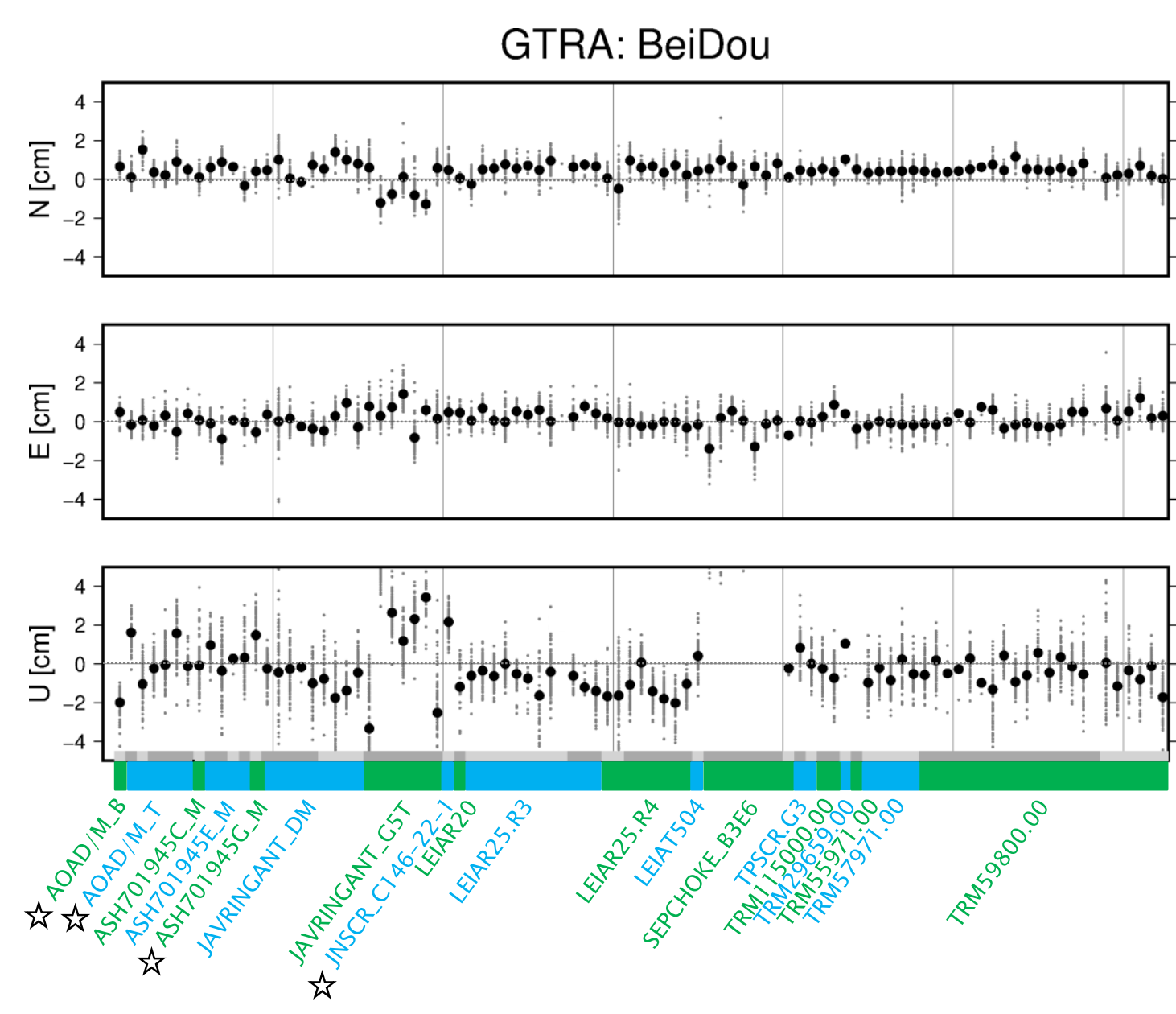

is Antennas without multi-GNSS calibrations
What are inter-system translation biases?

The inter-system translation bias, also called GNSS translation bias (GTRA), are equivalent to the difference between a GPS and a BeiDou only coordinate solution. If the receiver and satellite pattern are fully consistent the difference should be zero. However, there might be additional issues which lead to coordinate differences.

\section{Discussion:}

The differences are mostly scattered around zero. The GTRA indicate that the BeiDou antenna pattern can be used together with GPS, GLONASS, and Galileo without altering the coordinates. Note that even if the BeiDou pattern would be added to the IGSR3 ANTEX file it will not be an official part of the Repro3.

Potential reasons for big differences between GPS and BeiDou:

- The stations are tracking only a subset of the BeiDou satellites

- Issues with the antenna calibrations or other issues 


\section{BeiDou: Phase variations}
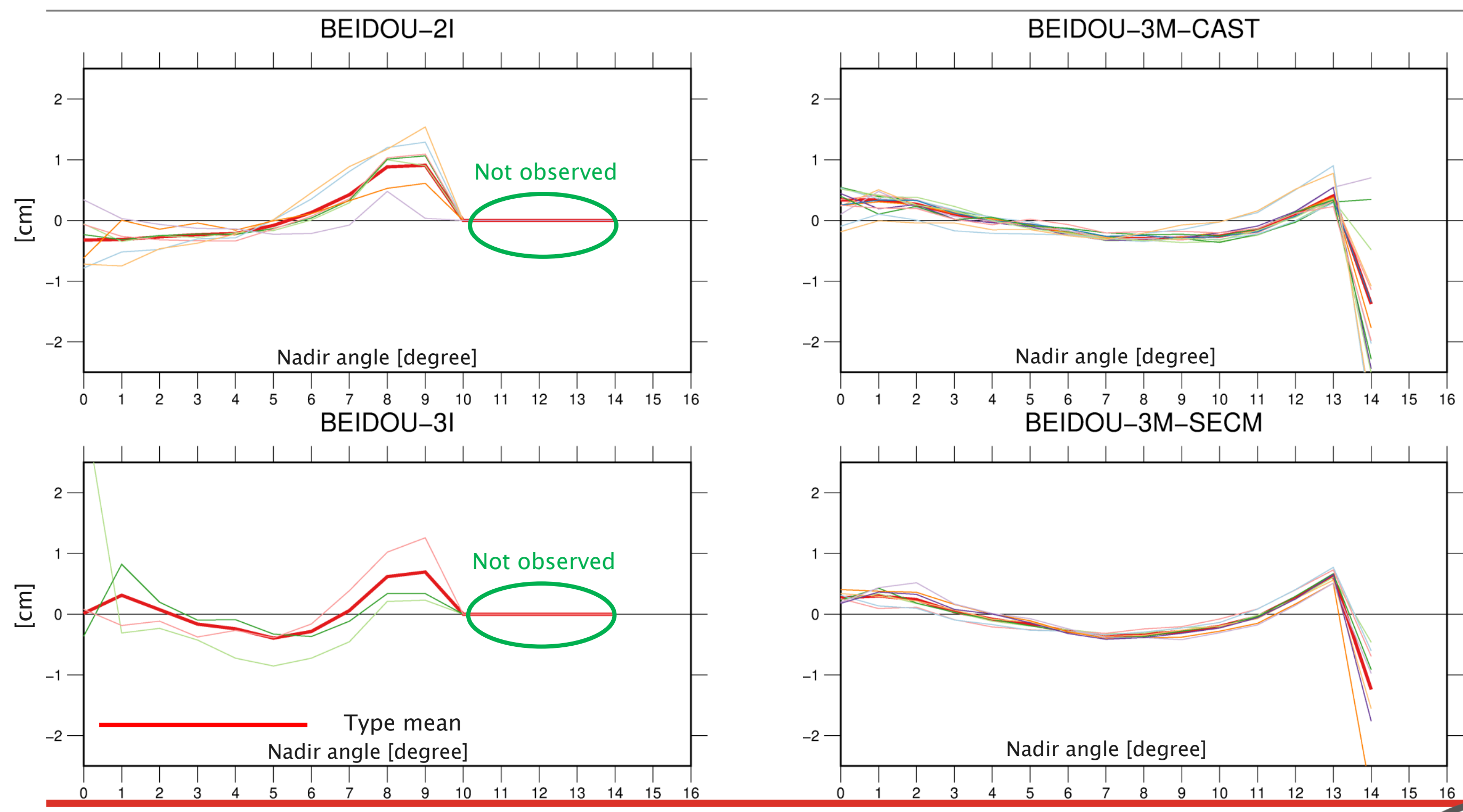


\section{QZSS (PCO corrections)}

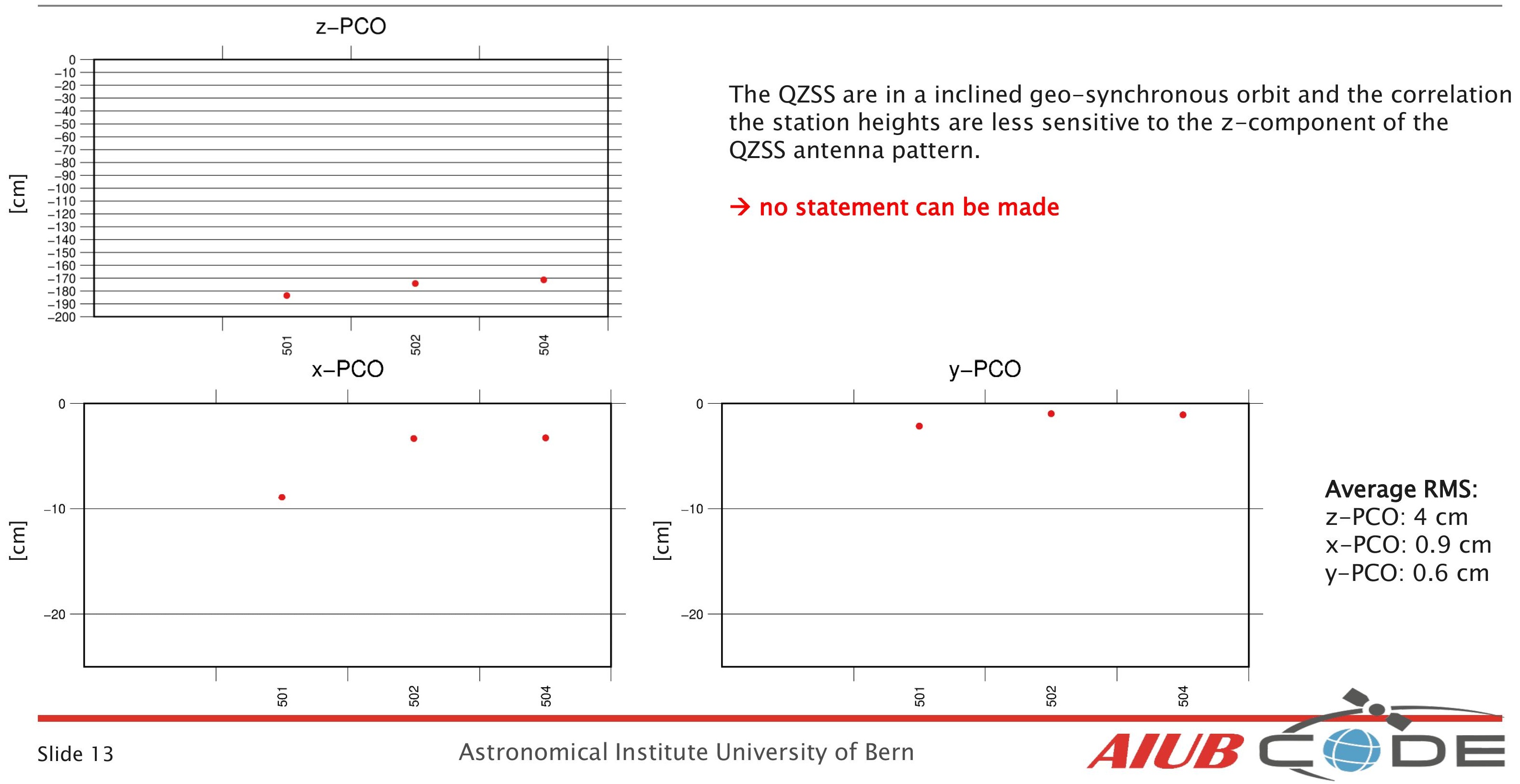




\section{Recommendation}

- Disclosed BLOCK IIIA pattern w.r.t. Repro3 compatible (except for G74)

- Further analysis of the horizontal component for BLOCK IIIA needed

- Add disclosed QZSS pattern to the Repro3 ANTEX. Station heights are less sensitive to the Z-PCO of QZS and IGSO satellites

- Use disclosed BeiDou pattern (compatible to the Repro3 scale). Further studies are needed for a potential contribution to a future ITRF

- Receiver calibration for all used frequencies is a must. The Repro3 ANTEX contains multi-GNSS robot calibrations from $\mathrm{Geo}++$ and chamber calibrations from the University of Bonn 